\title{
DISCOVERY OF A COOL, MASSIVE, AND METAL-RICH DAZ WHITE DWARF
}

\author{
A. Gianninas, P. Dufour and P. Bergeron \\ Département de Physique, Université de Montréal, C.P. 6128, Succ. Centre-Ville, \\ Montréal, Québec, Canada, H3C 3J\%. \\ gianninas, dufourpa, bergeron@astro.umontreal.ca
}

\begin{abstract}
We report the discovery of a new metal-rich DAZ white dwarf, GD 362. High signal-to-noise optical spectroscopy reveals the presence of spectral lines from hydrogen as well as Ca I, Ca II, Mg I, and Fe I. A detailed model atmosphere analysis of this star yields an effective temperature of $T_{\text {eff }}=9740 \pm 50 \mathrm{~K}$, a surface gravity of $\log g=9.12 \pm 0.07$, and photospheric abundances of $\log \mathrm{Ca} / \mathrm{H}=$ $-5.2 \pm 0.1, \log \mathrm{Mg} / \mathrm{H}=-4.8 \pm 0.1$, and $\log \mathrm{Fe} / \mathrm{H}=-4.5 \pm 0.1$. White dwarf cooling models are used to derive a mass of $1.24 M_{\odot}$ for GD 362, making it the most massive and metal-rich DAZ uncovered to date. The problems related to the presence of such large metal abundances in a nearby $(d \sim 25 \mathrm{pc})$ white dwarf in terms of an accretion scenario are briefly discussed.
\end{abstract}

Subject headings: stars : individual (GD 362) - stars : abundances - white dwarfs

\section{INTRODUCTION}

The most common type of white dwarfs are those that show hydrogen absorption lines in their optical spectra, the DA stars. A fraction of these, the so-called DAZ stars, also display absorption features from heavier elements, most notably the $\mathrm{Ca}$ II $\mathrm{H}$ and $\mathrm{K}$ lines. A recent spectroscopic survey at high dispersion of about $120 \mathrm{cool}\left(T_{\text {eff }}<10,000 \mathrm{~K}\right) \mathrm{DA}$ stars by Zuckerman et al. (2003) reveals that $\sim 25 \%$ of the objects in their sample show metallic features. The DAZ phenomenon thus seems much more common than previously believed (Billères et al. 1997). The origin of these metals in the atmospheres of DAZ stars is still poorly understood, however. Indeed, as most DAZ stars are comparatively cool and old, it is expected that all elements heavier than hydrogen would have settled rather quickly at the bottom of the atmosphere due to the strong gravitational field present in these stars. 
Therefore, any metals found in the atmosphere must be provided by an exterior source, such as the interstellar medium or even comets. We refer the reader to Zuckerman et al. (2003) for a more detailed discussion of the various theories that have been put forth to account for the presence of metals in DAZ stars.

We have recently undertaken a spectroscopic survey of white dwarfs drawn from the catalog of McCook \& Sion (1999). Our aim is to derive the atmospheric parameters for all stars, as well as to confirm the spectroscopic classifications given in the catalog using modern CCD spectroscopy. As part of this survey, we have discovered that GD 362 (WD 1729+371, $\mathrm{PG}$ ) is a unique DAZ white dwarf, with metallic features stronger than in any other member of its class, the Ca II H and $\mathrm{K}$ lines in particular. GD 362 had initially been classified as a DA white dwarf by Greenstein (1980) with a note that it could possibly constitute a composite spectrum or a dK subdwarf in a common proper motion binary system. It was later rediscovered in the Palomar-Green survey (Green et al. 1986) where it was classified $s d$, which does not imply a subdwarf type, but rather "a lower signal-to-noise observation in which two or three Balmer absorption lines of moderate gravity are visible"

We present in this Letter our spectroscopic data for GD 362 in which, besides the usual hydrogen Balmer lines, we have identified spectral lines of Ca I, Ca II, Mg I and Fe I. By fitting the optical spectrum with a grid of synthetic spectra appropriate for these stars, we determine the effective temperature and surface gravity of GD 362, as well as the abundances of all the elements observed in this star.

\section{FITTING PROCEDURE AND SYNTHETIC SPECTRA}

\subsection{Observations}

The high signal-to-noise (S/N 110 per pixel) optical spectrum of GD 362 was obtained over the course of 2 nights using the Steward Observatory $2.3 \mathrm{~m}$ telescope equipped with the Boller \& Chivens spectrograph. The 4.5 arcsec slit together with the $600 \mathrm{l} / \mathrm{mm}$ grating blazed at $\lambda 3568$ in first order provided a spectral coverage from about 3000 to $5250 \AA$ at a resolution of $\sim 6 \AA \mathrm{FWHM}$. The spectra were reduced using standard IRAF packages. The spectrum is displayed in Figure 1 where the major spectral features are identified, namely Ca I, Ca II, Mg I, and Fe I lines. We also show for comparison the optical spectrum of G74-7, discovered by Lacombe et al. (1983), which was the only DAZ star known until the more recent discoveries of G238-44 (Holberg et al. 1997), G29-38 (Koester et al. 1997), and those reported by Zuckerman et al. (2003). The comparison is most striking when we examine in both spectra the relative strengths of the $\mathrm{H}$ and $\mathrm{K}$ lines of Ca II at $3933.66 \AA$ and $3968.67 \AA$, 
respectively. It is worth noting that the region blueward of $\sim 3800 \AA$ is heavily blanketed by Fe I lines.

\subsection{Fitting Procedure}

The technique for fitting the spectrum is similar to that described at length by Liebert et al. (2004). First the continuum used to normalize the spectrum is set by fitting a model spectrum to the data, allowing for a possible wavelength shift, a zero point offset, and higher order terms in $\lambda$ (up to $\lambda^{3}$ ). The purpose of this procedure is simply to obtain a function that defines best the continuum of the observed spectrum. Once the spectrum has been normalized properly, the atmospheric parameters and chemical abundances are obtained from a grid of model spectra, convolved with a Gaussian profile at $6 \AA$ FWHM, using the nonlinear least-squares method of Levenberg-Marquardt (Press et al. 1986). This minimization technique also provides formal uncertainties of the fitted parameters through the covariance matrix (see Bergeron et al. 1992, for details).

\subsection{Synthetic Spectra}

For the computation of our synthetic spectra, we adopt a grid of LTE model atmospheres with a pure hydrogen composition similar to that described in Liebert et al. (2004). We thus make the assumption that the metals have no effect on the atmospheric structure and include them only in the calculation of the emergent fluxes; this assumption will be tested subsequently. Atomic partition functions have been calculated by summing explicitly over the bound states taken directly from TOPBASE. Central wavelengths of the transitions, $g f$ values, energy levels, and damping constants (radiative, Stark, and van der Waals) have been extracted from the GFALL linelist of Kurucz (http://kurucz.harvard.edu/LINELISTS.html).

Since our preliminary analysis of GD 362 (Gianninas et al. 2005) revealed that it was a high surface gravity DAZ star, we restrict our model grid calculations to high values of $\log g$. We first begin with the determination of the calcium abundance since calcium produces the most prominent features in the spectrum. Our model grid covers a range of $-5.7 \leq \log \mathrm{Ca} / \mathrm{H} \leq-4.8$ in steps of 0.3 dex, effective temperatures in the range $9000 \leq$ $T_{\text {eff }} \leq 10,400 \mathrm{~K}$ in steps of $200 \mathrm{~K}$, and surface gravities in the range $8.75 \leq \log g \leq 9.50$ in steps of 0.25 dex. With the calcium abundance fixed, we proceed to determine in a similar

way the magnesium and iron abundances in turn, using spectra with magnesium abundances in the range $-5.6 \leq \log \mathrm{Mg} / \mathrm{H} \leq-4.4$ in steps of $0.4 \mathrm{dex}$, and iron abundances in the range 
$-5.0 \leq \log \mathrm{Fe} / \mathrm{H} \leq-4.4$ in steps of 0.2 dex. At each step, the determination of the effective temperature and surface gravity is refined.

\subsection{Atmospheric Parameter and Abundance Determinations}

Before undertaking our analysis of GD 362, we wished to test our grid of synthetic spectra, and more importantly our fitting procedure. With this in mind, we fitted the optical spectrum of G74-7 analyzed by Billères et al. (1997) who derived $T_{\text {eff }}=7260 \pm 40 \mathrm{~K}$, $\log g=8.03 \pm 0.07$, and $\log \mathrm{Ca} / \mathrm{H}=-8.8 \pm 0.1$. Our own analysis of the same spectrum yields $T_{\text {eff }}=7300 \pm 60 \mathrm{~K}, \log g=8.02 \pm 0.11$, and $\log \mathrm{Ca} / \mathrm{H}=-8.9 \pm 0.3$, in excellent agreement with the results of Billères et al.

Our best fit to the spectrum of GD 362 is displayed in Figure 2. The atmospheric parameters and corresponding uncertainties are $T_{\text {eff }}=9740 \pm 50 \mathrm{~K}, \log g=9.12 \pm 0.07$, $\log \mathrm{Ca} / \mathrm{H}=-5.2 \pm 0.1, \log \mathrm{Mg} / \mathrm{H}=-4.8 \pm 0.1$, and $\log \mathrm{Fe} / \mathrm{H}=-4.5 \pm 0.1$. We are able to obtain a very good fit to the observed spectrum albeit not a perfect one. First, we notice that although the $\mathrm{H}$ and $\mathrm{K}$ lines of $\mathrm{Ca}$ II are extremely well reproduced, the Ca I lines at 4227 and $4585 \AA$ are predicted too strong. We believe that this is caused by an incorrect $\mathrm{Ca}$ II/Ca I ratio which could be due either to an erroneous electron density or partition function values obtained from TOPBASE. Second, the Fe I lines in the bluest portion of the spectrum are not reproduced perfectly. Some of the atomic data for iron is derived from theoretical calculations and is more uncertain than values determined experimentally. We therefore suggest that our derived iron abundance is at worse an upper limit.

A posteriori, we computed a blanketed model atmosphere in which we took into account the presence of metals at our derived abundances. The synthetic spectrum calculated from this model is identical to that computed here from our pure hydrogen models, validating our initial assumption that the metals play a negligeable role in the determination of the atmospheric structure.

\section{DISCUSSION}

Using the evolutionary models of Fontaine et al. (2001) for DA white dwarfs, we derive a mass of $1.24 M_{\odot}$. Our derived atmospheric parameters thus make GD 362 the most metalrich and massive DAZ white dwarf studied thus far, and somewhat hotter than the majority of DAZ stars discovered by Zuckerman et al. (2003). In Figure 3, we show the calcium abundance as a function of effective temperature for the white dwarfs from Zuckerman et 
al. (2003). We see from its location in Figure 3 how unique GD 362 truly is. In fact, if we compare with the values listed in Table 4 of Zuckerman et al. (2003), the iron and magnesium abundances are nearly solar!

A comparison of our calcium abundance determination for GD 362 with those of other DAZ stars from Zuckerman et al. (2003) with similar effective temperatures reveals that the abundance observed in GD 362 is at least a factor of $10^{3}$ higher (see Fig. 3). Since the mass of GD 362 is also higher than average, we need to verify whether this is the reason for the unusual observed photospheric abundances. To do so, we compare the expected steady-state calcium abundance in a massive star at $1.2 M_{\odot}$ to that of a normal star with a mass of 0.6 $M_{\odot}$. The steady-state abundance, $X_{\mathrm{ss}}$, is given by equation (4) of Dupuis et al. (1993),

$$
X_{\mathrm{ss}}=\frac{\theta \dot{M}}{\Delta M_{\mathrm{cz}}},
$$

where $\theta$ is the diffusion time scale of calcium at the bottom of the hydrogen convection zone, $\dot{M}$ is the accretion rate of that element, and $\Delta M_{\mathrm{cz}}$ is the mass of the convection zone. If we assume the Bondi-Hoyle accretion rate, then $\dot{M}$ is proportional to $M^{2}$, where $M$ is the mass of the white dwarf. Then the expected relative abundance of calcium between a 1.2 and a $0.6 M_{\odot}$ white dwarf can be expressed as

$$
\frac{X_{1.2}}{X_{0.6}} \propto \frac{\theta_{1.2}}{\theta_{0.6}} \frac{M_{1.2}^{2}}{M_{0.6}^{2}} \frac{\Delta M_{\mathrm{cz}, 0.6}}{\Delta M_{\mathrm{cz}, 1.2}}
$$

where we assume that both stars are moving with the same velocity through an interstellar medium of similar density. Using values of $\theta$ and $\Delta M_{\mathrm{cz}}$ generously provided to us by G. Fontaine (private communication), we obtain that

$$
\frac{X_{1.2}}{X_{0.6}} \propto \frac{1}{36} \cdot 4 \cdot 8.5 \approx 1
$$

We see that even though a more massive star accretes at a higher rate and has a thinner convection zone - implying a smaller dilution factor, the diffusion time scale at the bottom of the hydrogen convection zone is so much shorter that comparable abundances are predicted.

Additionally, the accretion model suffers from the fact there is little interstellar matter within 100 pc from the Sun, as Aanestad et al. (1993) aptly pointed out. The distance to GD 362 can be estimated in two ways. First, the $T_{\text {eff }}$ and $\log g$ values inferred for GD 362 together with the photometric calibration of Bergeron et al. (1995) yield an absolute visual magnitude of $M_{V}=14.31$ ( or $M_{B}=14.48$ ), which combined with the photographic 
magnitude of $B_{\mathrm{ph}}=16.15$ taken from Green et al. (1986), yields a distance estimate of $\sim 22$ pc. Alternatively, we can use the relation between the monochromatic fluxes from our optical spectrum, $f_{\lambda}$, and the theoretical Eddington fluxes, $H_{\lambda}$, given by

$$
f_{\lambda}=4 \pi(R / D)^{2} H_{\lambda}
$$

where $R$ and $D$ are the radius of the star and its distance from Earth, respectively, to obtain an independent distance estimate of $\sim 26 \mathrm{pc}$, consistent with our previous estimate. In either case, GD 362 seems to be well within the local bubble and far from any high concentrations of interstellar matter. We are therefore at odds to explain the very high metal abundances observed.

Another possibility would be that GD 362 is an unresolved degenerate binary composed of a normal DA star and a helium-rich DZ star of comparable luminosities. If this were the case, however, the intrinsic absorption features of each star would be even stronger than observed here, because of the line dilution factor, and the effective temperature inferred from the Balmer lines would thus be grossly underestimated (Balmer lines get stronger with increasing $T_{\text {eff }}$ in this temperature range). In such unresolved systems, the slope of the theoretical spectrum is incompatible with that of the observed spectrum (see, e.g., Bergeron et al. 1990). We see on the contrary from Figure 2 that the slope of the theoretical spectrum is in perfect agreement with the observed slope of GD 362, consistent with the presence of a single star.

It is perhaps not too suprising that GD 362 had not been classified as a DAZ white dwarf by Greenstein (1980) or Green et al. (1986). The very strong Ca II H and K lines seen on their photographic spectrum, in addition to the narrow Balmer lines, have led these investigators to classify GD 362 as composite or as a lower gravity object. This raises the interesting possibility that many such objects exist and have been overlooked both in the past and in modern surveys such as the Sloan Digital Sky Survey.

We thank Gilles Fontaine and Pierre Brassard for useful discussions. We would also like to thank the director and staff of Steward Observatory for the use of their facilities, as well as the referee for useful suggestions. This work was supported in part by the NSERC Canada and by the FQRNT (Québec). 


\section{REFERENCES}

Aanestad, P. A., Kenyon, S. J., Hammond, G. L., \& Sion, E. M. 1993, AJ, 105, 1033

Bergeron, P., Greenstein, J. L., \& Liebert, J. 1990, ApJ, 361, 190

Bergeron, P., Saffer, R. A., \& Liebert, J. 1992, ApJ, 394, 228

Bergeron, P., Wesemael, F., \& Beauchamp, A. 1995, PASP, 107, 1047

Billères, M., Wesemael., F., Bergeron, P., \& Beauchamp, A. 1997, ApJ, 488, 368

Dupuis, J., Fontaine, G., Pelletier, C., \& Wesemael F. 1993, ApJS, 84, 73

Fontaine, G., Brassard, P., \& Bergeron, P. 2001, PASP, 113, 409

Gianninas, A., Bergeron, P., \& Dufour, P. 2005, in 14th European Workshop on White Dwarfs, ASP Conf. Series, vol. 999, eds. D. Koester \& S. Moehler, in press

Green, R. F., Schmidt, M., \& Liebert, J. 1986, ApJS, 61, 305

Greenstein, J. L. 1980, ApJ, 242, 738

Holberg, J. B., Barstow, M. A., \& Green, E. M. 1997, ApJ, 474, L127

Koester, D., Provencal, J., \& Shipman, H. L. 1997, A\&A, 230, L57

Lacombe, P., Wesemael, F., Fontaine, G., \& Liebert, J. 1983, ApJ, 272, 660

Liebert, J., Bergeron, P., \& Holberg, J. B. 2004, ApJS, in press

McCook, G. P., \& Sion, E. M. 1999, ApJS, 121, 1

Press, W. H., Flannery, B. P., Teukolsky, S. A., \& Vetterling, W. T. 1986, Numerical Recipes (Cambridge: Cambridge University Press)

Zuckerman, B., Koester, D., Reid, I. N., \& Hünsch, M. 2003, ApJ, 596, 477 
Fig. 1.- Flux calibrated optical spectra of G74-7 (top) and GD 362 (bottom), normalized to unity at $4500 \AA$, and offset from each other by a factor of 0.7 . The major metallic features in the spectrum of GD 362 are indicated by tick marks. In both cases, H $\epsilon$ is blended with Ca II $\lambda 3969$.

Fig. 2.- Our best fit (bottom) to the observed spectrum of GD 362 (top). The atmospheric parameters obtained from this analysis are indicated along with the mass derived from the evolutionary models of Fontaine et al. (2001).

Fig. 3.- Calcium abundances, or upper limits, as a function of effective temperature for the white dwarfs taken from Tables 1 and 2 of Zuckerman et al. (2003). The open circles mark objects where the Ca II K line was detected, while the crosses denote white dwarfs with only upper limit determinations. The filled circle represents GD 362. 


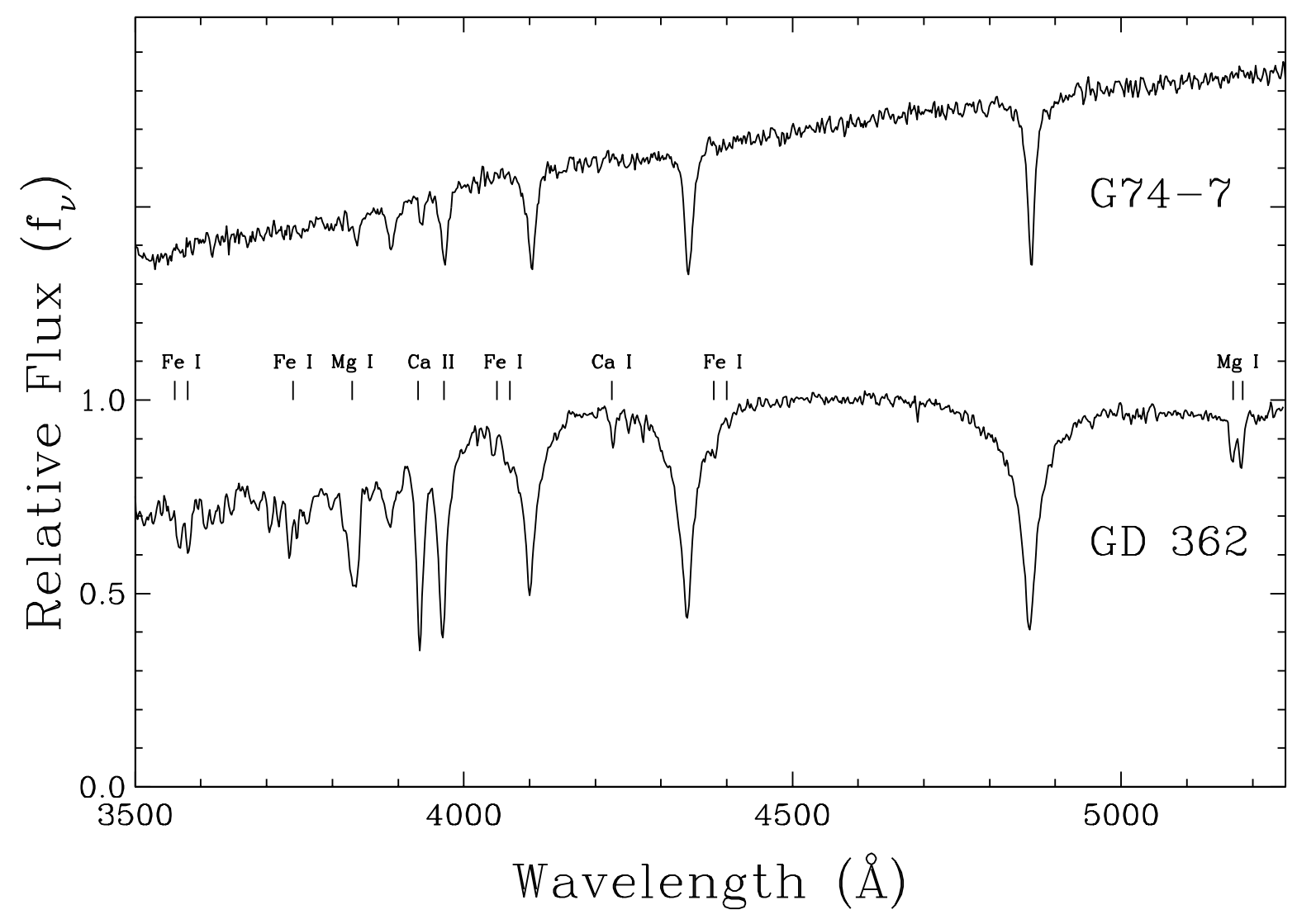

Figure 1 


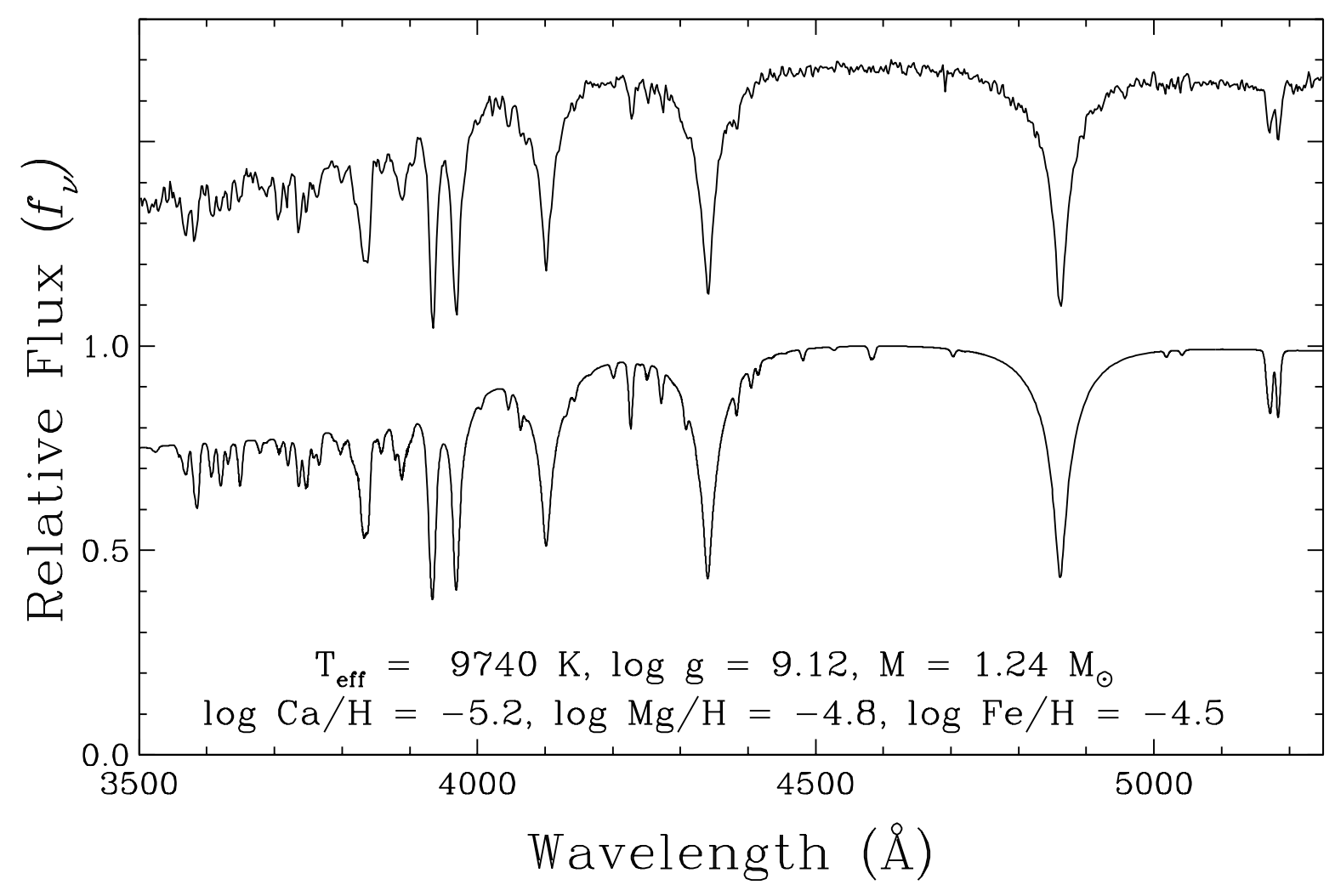

Figure 2 


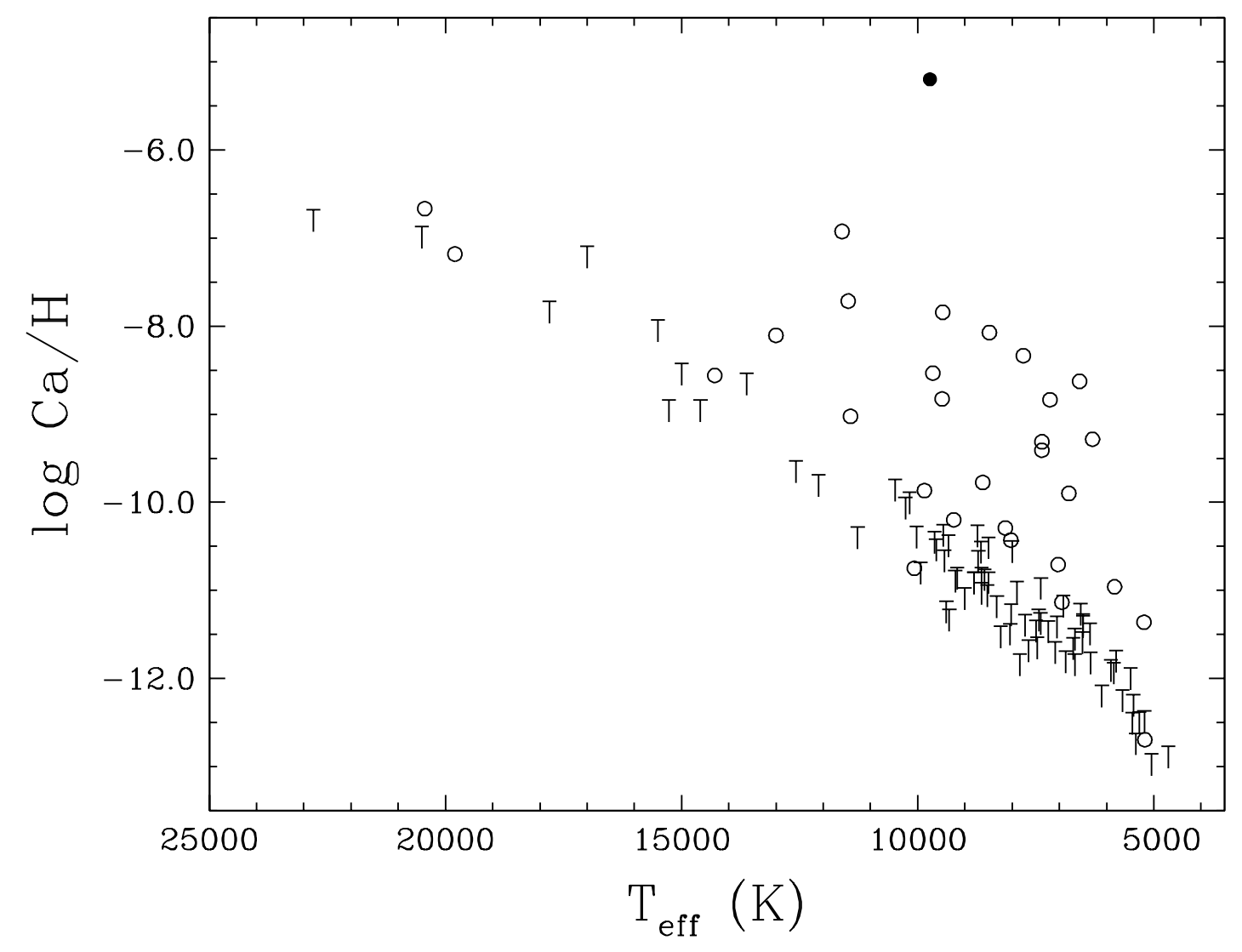

Figure 3 